\title{
Super-Massive Neutron Stars and Compact Binary Millisecond Pulsars
}

\author{
Manuel Linares $* a b$ \\ ${ }^{a}$ Departament de Física, EEBE, Universitat Politècnica de Catalunya, Av. Eduard Maristany 16, \\ E-08019 Barcelona, Spain. \\ ${ }^{b}$ Institute of Space Studies of Catalonia (IEEC), E-08034 Barcelona, Spain. \\ E-mail: manuel.1inares@upc.edu
}

\begin{abstract}
The maximum mass of a neutron star has important implications across multiple research fields, including astrophysics, nuclear physics and gravitational wave astronomy. Compact binary millisecond pulsars (with orbital periods shorter than about a day) are a rapidly-growing pulsar population, and provide a good opportunity to search for the most massive neutron stars. Applying a new method to measure the velocity of both sides of the companion star, we previously found that the compact binary millisecond pulsar PSR J2215+5135 hosts one of the most massive neutron stars known to date, with a mass of $2.27 \pm 0.16 \mathrm{M}_{\odot}$ (Linares, Shahbaz \& Casares, 2018). We reexamine the properties of the $0.33 \mathrm{M}_{\odot}$ companion star, heated by the pulsar, and argue that irradiation in this "redback" binary is extreme yet stable, symmetric and not necessarily produced by an extended source. We also review the neutron star mass distribution in light of this and more recent discoveries. We compile a list of all (nine) systems with published evidence for supermassive neutron stars, with masses above $2 \mathrm{M}_{\odot}$. We find that four of them are compact binary millisecond pulsars (one black widow, two redbacks and one redback candidate). This shows that compact binary millisecond pulsars are key to constraining the maximum mass of a neutron star.
\end{abstract}

Multifrequency Behaviour of High Energy Cosmic Sources - XIII - MULTIF2019

3-8 June 2019

Palermo, Italy

${ }^{*}$ Speaker. 


\section{Introduction: the maximum neutron star mass}

The maximum mass that a neutron star (NS) can sustain, $\mathrm{M}_{N S}^{\max }$, has important implications across multiple research fields, including astrophysics, nuclear physics and gravitational wave (GW) astronomy. From a purely observational viewpoint, the distinction between neutron stars and stellar-mass black holes in binary systems is often based on the mass of the primary star, which is classified as a black hole if its mass exceeds $\mathbf{M}_{N S}^{\max }$ [1]. The birth masses of NSs depend on the still uncertain physical mechanisms taking place in supernovae, when the proto-NS is being formed during the gravitational collapse of a massive star [2]. Later evolution in an interacting binary can significantly increase the mass of a NS via accretion, as well as decrease its spin period and surface magnetic field, during the so-called recycling stage [3]. More recently, the maximum NS mass has regained interest in the context of double neutron star mergers and the associated GW emission [4]. Simply put, what is left after two neutron stars merge depends on their masses before coalescing, and on the maximum mass that a neutron star can hold [e.g., 5]. It is also important to know $\mathrm{M}_{N S}^{\max }$ well enough to distinguish between massive NSs and light black holes, in the new era of GW events from binary mergers.

The maximum mass of a NS depends on the compressibility of its core, and on the equation of state (EoS, the relation between pressure and density) at densities close to and above nuclear saturation density $\left(\rho_{\text {nис }} \simeq 3 \times 10^{14} \mathrm{~g} \mathrm{~cm}^{-3}\right.$ ). Relatively incompressible matter (described by "stiff" EoSs) is needed to support more massive NSs. Microscopically, the EoS in the core and $\mathrm{M}_{N S}^{\max }$ are set by the nucleon-nucleon interactions in the central parts of a NS, which remain uncertain despite six decades of research [6]. Therefore, the maximum mass of a NS has a critical impact on nuclear physics, as it places direct constraints on the EoS of matter at supra-nuclear densities $[7 ; 8 ; 9]$.

The NS mass distribution has evolved as new types of NSs in binaries were discovered and their masses measured. The majority of NS mass measurements come from rotation-powered radio pulsars in binary systems (299 listed at the ATNF catalog, v1.60; [10]), most of which are binary millisecond pulsars (MSPs, 249 catalogued with spin periods shorter than $30 \mathrm{~ms}$; see also [11]). Two decades ago, the first measurements suggested a single and narrow NS mass distribution near $1.4 \mathrm{M}_{\odot}\left[1.35 \pm 0.04 \mathrm{M}_{\odot}\right.$ from 26 pulsars, mostly in double NSs; 12]. In 2010 and 2013, NSs with masses close to $2 \mathrm{M}_{\odot}$ were found in binary pulsars with white dwarf (WD) companions [13; 14]. These were accurate measurements ( $\simeq 2 \% 1-\sigma$ errors; see Table 1$)$ obtained with different methods, and they established that NSs can be as massive as $2 \mathrm{M}_{\odot}$. In 2011, van Kerkwijk et al. [15] found evidence for a NS with more than two Solar masses, $2.40 \pm 0.12 \mathrm{M}_{\odot}$, in one of only four compact binary MSPs known in the Galactic field at the time (see Section 2 for definition and details).

Recently, Özel \& Freire [9] compiled NS mass (and radius) measurements, discussing in depth the NS mass distribution. Thanks to these and other previous studies $[7 ; 8 ; 16]$, we know now that NS masses span a wide range, between 1.2 and at least $2 \mathrm{M}_{\odot}$. However, compact binary MSPs (black-widows and redbacks) have not been included in most global studies of the NS mass distribution, perhaps due to their past scarcity or in order to avoid the uncertainties in correcting for the strong irradiation of the companion star by the pulsar wind. Here, we review the most massive NSs presently known in light of recent findings and including compact binary MSPs, which we argue are key to constraining $\mathbf{M}_{N S}^{\max }[15 ; 17 ; 18 ; 19 ; 20]$. 


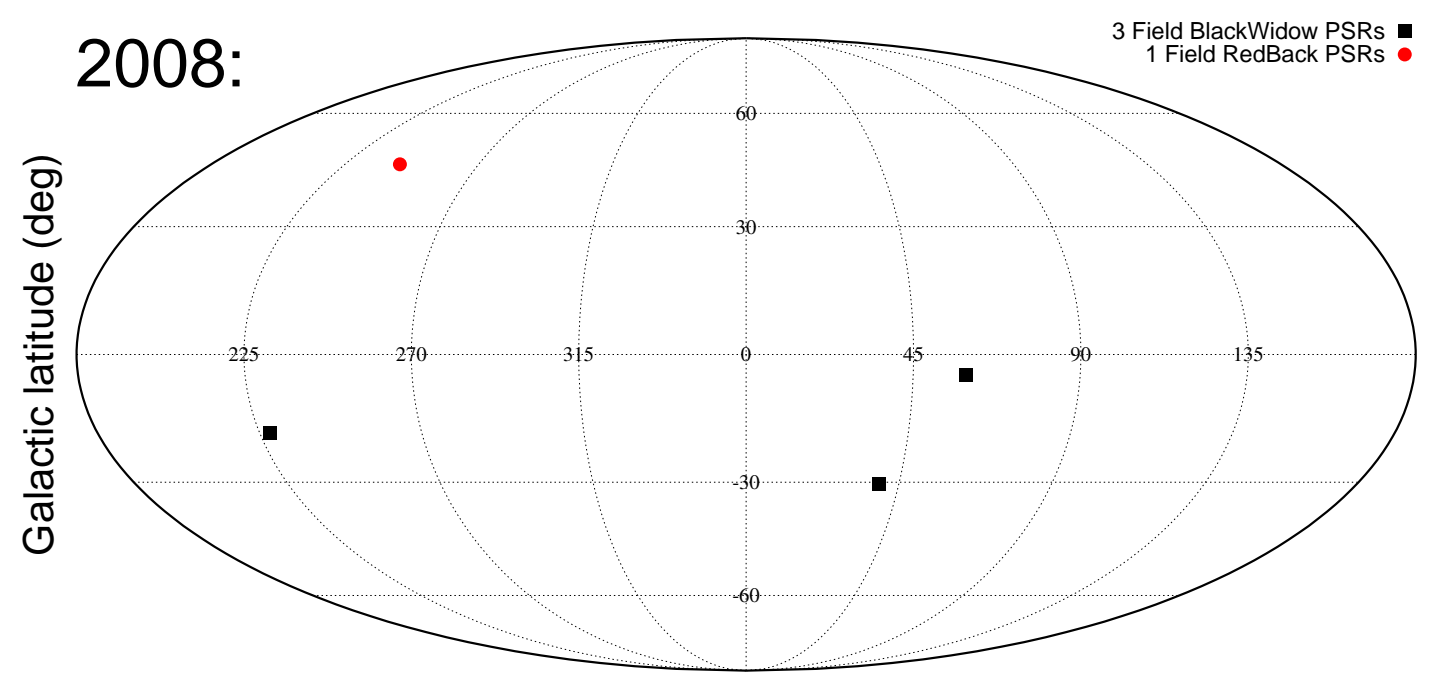

Galactic longitude (deg)

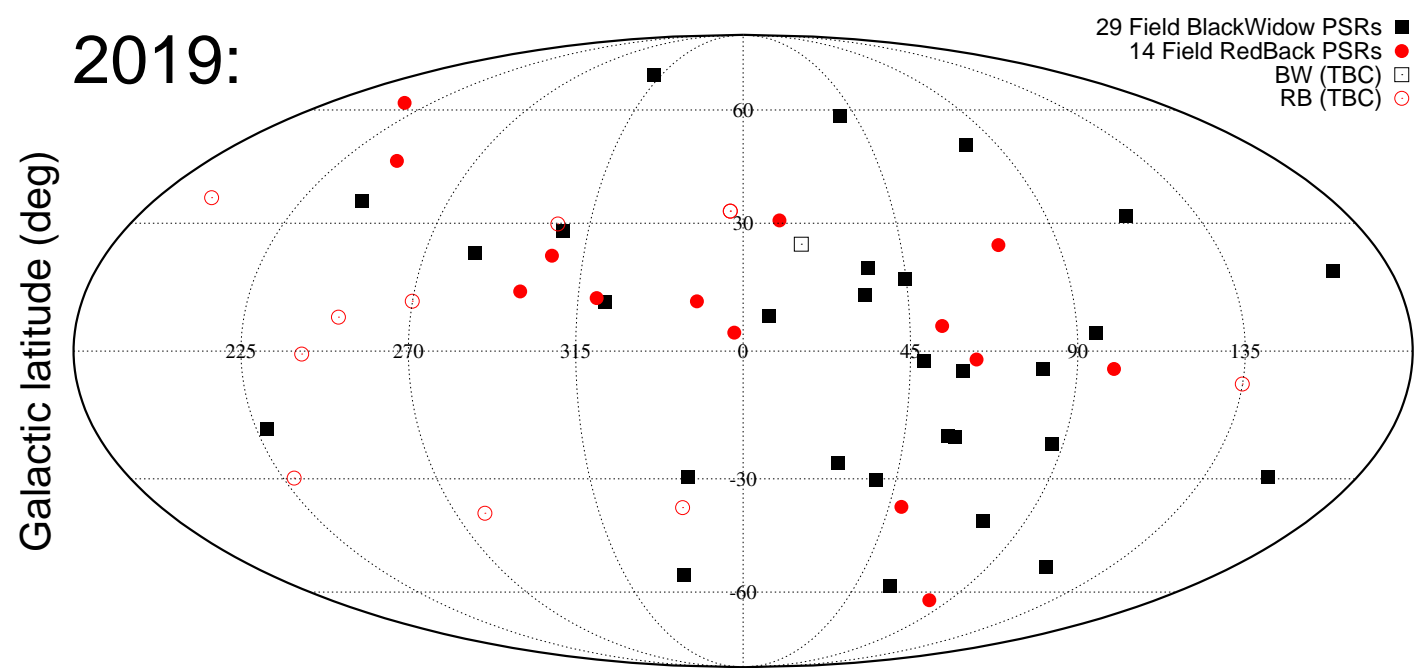

Galactic longitude (deg)

Figure 1: All-sky map (Mollweide projection) showing the Galactic coordinates of the compact binary MSPs known in 2008 (top, 4 systems) and 2019 (bottom, 43 confirmed +11 candidates). Black squares and red circles show black-widow and redback MSPs, respectively. Open symbols show candidate systems, where the detection of a radio/gamma-ray pulsar has not been reported yet.

\section{A spider revolution: millisecond pulsars in compact binaries}

Compact binary MSPs have orbital periods shorter than about a day and form two distinct populations, with either a low-mass main-sequence companion star in the systems known as "redbacks" (RBs; $\mathrm{M}_{c} \gtrsim 0.1 \mathrm{M}_{\odot}$ ), or a very low-mass "ultra-light" companion in those known as "black widows" (BWs; $\mathrm{M}_{c} \sim 0.01 \mathrm{M}_{\odot}$ ). They have been nicknamed after cannibalistic spiders because of the destructive effect that the pulsar wind may have on its companion, and are sometimes referred 
collectively as "spiders". Before the launch of the Fermi gamma-ray telescope in 2008, only four compact binary MSPs were known in the field of the Galaxy, as shown in Figure 1 (top). These exclude MSPs in globular clusters, which now contain 12/18 RBs/BWs [21] but are typically unsuited for dynamical mass measurements. Pulsars are relatively bright sources at $\mathrm{GeV}$ energies, the band where Fermi's large area telescope (LAT, $\sim 0.1-100 \mathrm{GeV}$; [22; 23]) is providing unprecedented sensitivity. Combined with targeted radio observing campaigns $[24 ; 25 ; 26 ; 27 ; 28 ; 29 ; 30 ; 31]$ as well as optical and multi-wavelength follow-up [32;33; 34; 35; 36; 37], this has turned Fermi-LAT into a true "pulsar discovery machine".

Surprisingly, many of these LAT-driven discoveries have revealed new compact binary MSPs, so their population has increased drastically over the past decade, from 4 to more than 40 (Figure 1, bottom). This is what we may call "a spider revolution": a new class of nearby ( $d \leqslant 3 \mathrm{kpc})$ and energetic (spin-down luminosity $\dot{E} \sim 10^{34}-10^{35} \mathrm{erg} \mathrm{s}^{-1}$ ) pulsars has emerged. Because most new spiders are also far from the Galactic plane, where interstellar extinction is low, they are well within reach of optical telescopes (especially redbacks, with brighter companion stars). This allows for spectroscopic studies of the companion star and dynamical measurements of the MSP mass, as detailed below. During a long (Gyr) formation phase with active mass transfer, spiders are thought to accrete mass from their companion stars, so that NSs in compact binary MSPs may be significantly more massive than they were at birth [38]. Thus, among other fields, this new MSP population is having a strong impact on $\mathrm{M}_{N S}^{\max }$ and the NS mass distribution.

\section{A 2.3 Solar-mass neutron star in PSR J2215+5135}

In 2014, we set out to measure the mass of a redback pulsar in a $4.14 \mathrm{hr}$ orbit, PSR J2215+5135, which had previous evidence for a massive NS and a strongly irradiated companion star [18; 39]. We observed the system with three optical telescopes, obtaining high signal-to-noise photometry and including the Gran Telescopio Canarias (GTC, 10.4-m diameter) in order to collect high-quality spectra throughout the orbit. Thanks to these GTC spectra we were able to find a new and subtle effect: among the absorption lines from the companion star's atmosphere, metallic lines trace the unheated "cold" side of the star and thus move at higher velocities than hydrogen lines, which are formed predominantly on the inner "hot" side of the star. In particular, we found that magnesium lines (MgI triplet at 5167-5184 $\AA$ ) move $10 \%$ faster than Balmer lines $(\mathrm{H} \beta, \gamma, \delta)$. This allowed us to find empirically and robustly the velocity of the center of mass of the star $\left(\mathrm{K}_{2}\right)$, a very important step to obtain reliable mass measurements in irradiated binary systems.

Furthermore, we measured the temperature of the star throughout the orbit using a suite of absorption lines and confirmed quantitatively the extreme irradiation of the inner side of the companion produced by the pulsar wind (from $\mathrm{T}_{\mathrm{N}}=5660_{-380}^{+260} \mathrm{~K}$ to $\mathrm{T}_{\mathrm{D}}=8080_{-280}^{+470} \mathrm{~K}$ ). Imposing these independent temperature constraints, we modeled jointly the light and radial velocity curves in order to obtain the orbital inclination $\left(\mathrm{i}=63.9^{\circ} \pm 2.5\right.$ ) and the masses of both stars. While our work was being completed, Romani et al. reported higher inclinations from independent dynamical studies of PSR J2215+5135 [40; 41]. Because their reported temperatures differ from ours and have no uncertainties, we concluded that accurate temperature constraints are critical to measure robustly masses and inclination. For the full details and discussion the reader is referred to Linares, Shahbaz \& Casares (2018, [19]). 


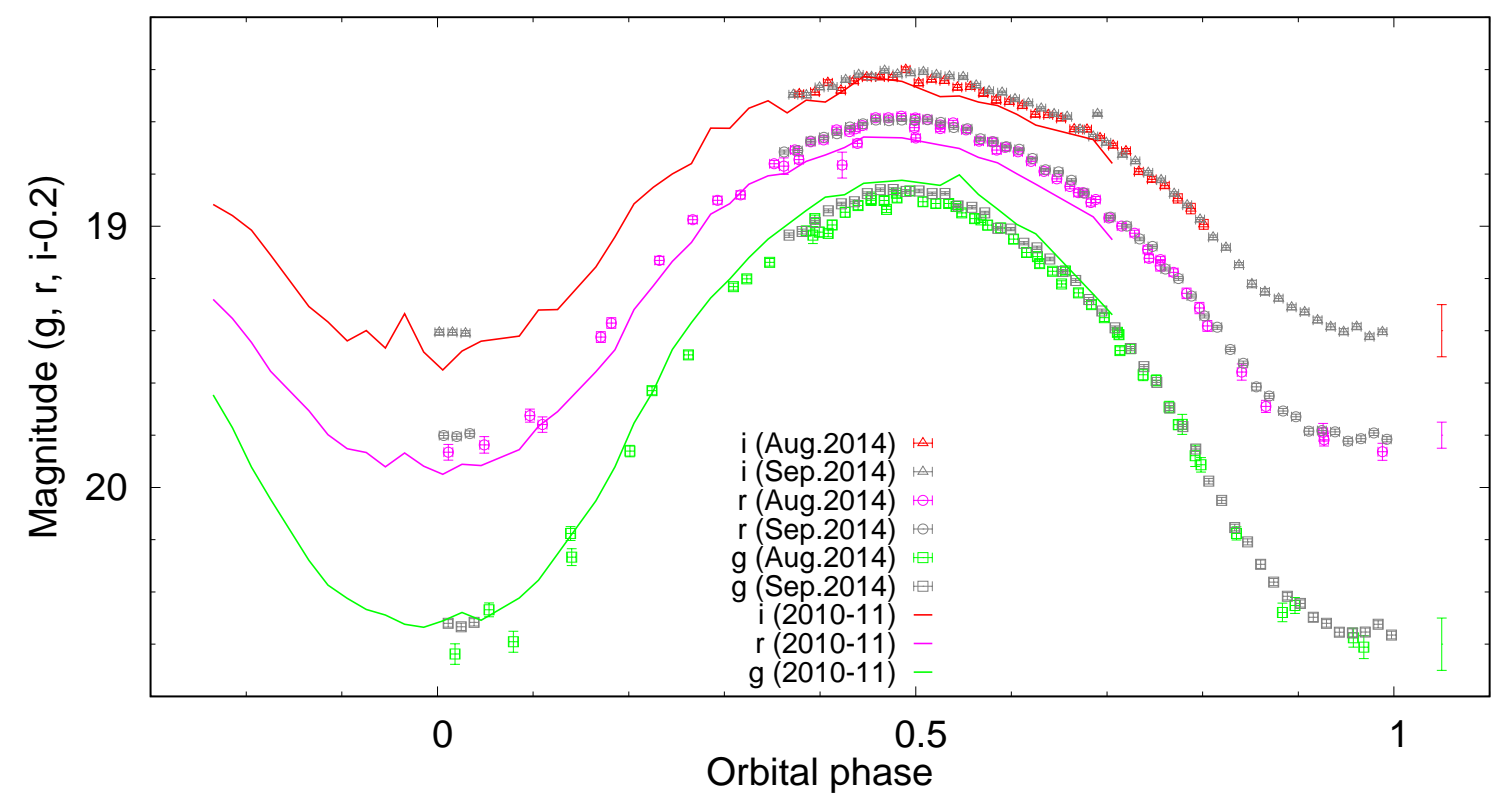

Figure 2: Optical light curves of PSR J2215+5135 taken during four different nights with the WHT and IAC80 telescopes on August (red triangles, magenta circles and green squares for the i, $\mathrm{r}$ and $\mathrm{g}$ bands, respectively) and September (gray symbols), 2014. Solid lines show the light curves measured by Schroeder \& Halpern in 2011 (3 years earlier, converted from BVR filters; [39]). The orbital light curves of this redback MSP are smooth (down to the shortest $60 \mathrm{~s}$ exposures) and stable on timescales of days to years (within the available data and its photometric precision, shown by the error bars on the right).

We thereby found one of the most massive NSs known to date in PSR J2215+5135, with $\mathrm{M}_{\mathrm{NS}}=2.27 \pm 0.16 \mathrm{M}_{\odot}$ [19]. A 2.3 Solar-mass neutron star rules out most currently proposed equations of state, casting doubt on the existence of exotic forms of matter in the core. It is worth stressing here that the optical light curves and spectra of PSR J2215+5135 are "well behaved", in the sense that they only show the orbital modulation typical of irradiated compact binaries. No flares, long-term variability or transient emission lines have been detected (see, e.g., multi-epoch light curves in Figure 2). We were able to fit jointly and satisfactorily these three-band light curves (g,r,i) as well as the two-species radial velocity curves (Balmer and MgI) assuming point-like irradiation by the pulsar wind, without any extended heating source (unlike previous work [41]). Furtermore, after carefully considering the uncertainty in the orbital phases, we did not find in our data significant asymmetry in the optical lightcurves of PSR J2215+5135 [39; 40]. Summarizing, in our optical study of this particular redback we found that even if heating/irradiation of the companion by the MSP is extreme: i) it is not variable, ii) it is not necessarily produced by an extended region and iii) it shows little or no asymmetry. We argue that this, together with our new empirical method to determine $\mathrm{K}_{2}$, makes the $2.3 \mathrm{M}_{\odot}$ measurement robust.

\section{Super-massive neutron stars: breaking the $2 \mathrm{M}_{\odot}$ barrier}

Figure 3 shows an updated compilation of 86 NS mass measurements, including 17 compact binary MSPs $([9 ; 20 ; 45]$, and references therein). This readily shows that redback and black widow 


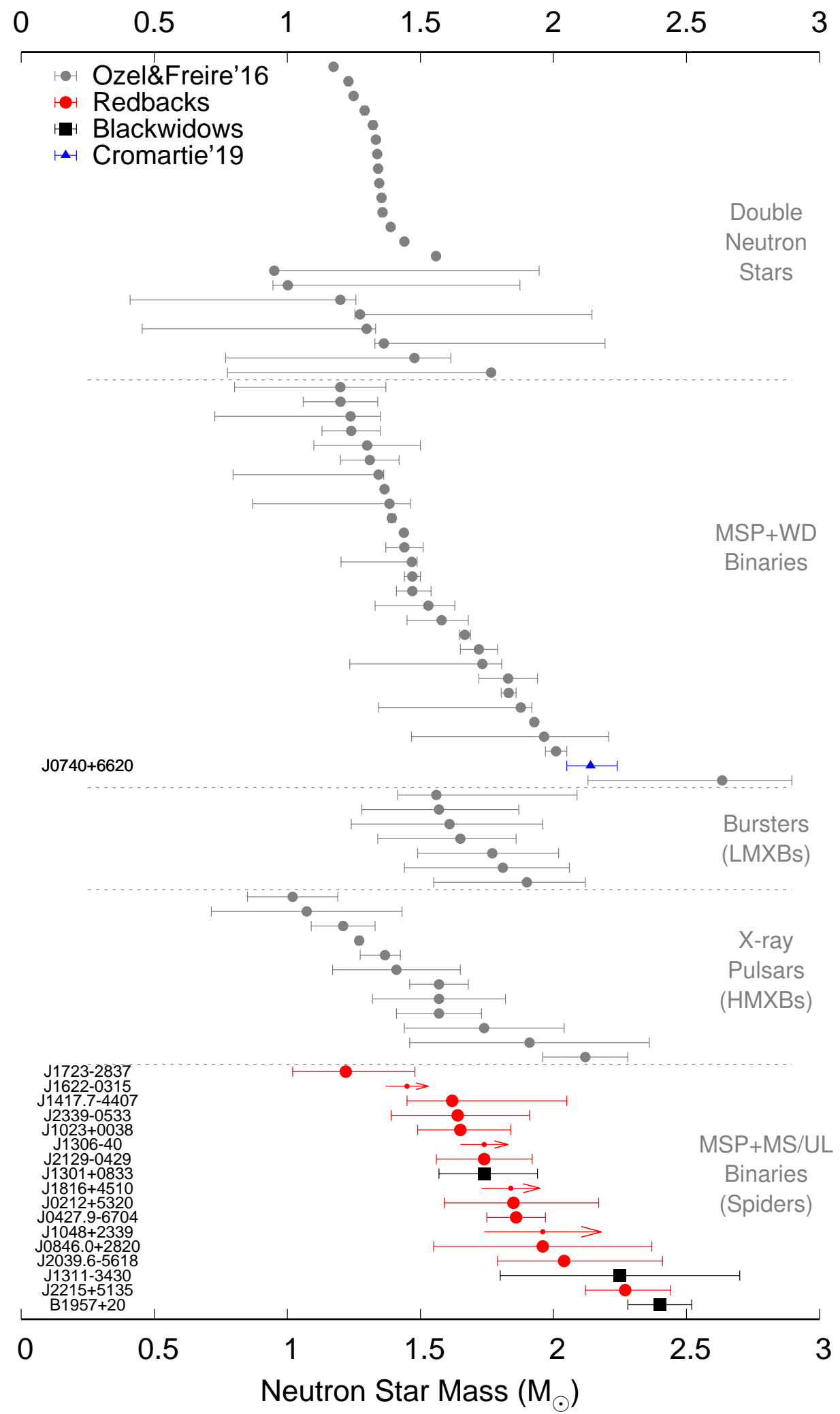

Figure 3: Masses of 17 compact binary MSPs (red circles and black squares, as indicated; arrows show lower limits on $\left.\mathrm{M}_{N S} ;[42 ; 43 ; 44 ; 19 ; 15 ; 20]\right)$. For comparison, we show 68 NS mass measurements compiled by Özel \& Freire ([9], gray circles; see references therein). Different types of binary NSs are noted with labels along the right side. The recent NS mass measured by Cromartie et al. [45] is shown in blue. 
MSPs (or "spiders") tend to push the NS mass range beyond the $2 \mathrm{M}_{\odot}$ limit. In Table 1 we collect those systems with NS mass measurements above $2 \mathrm{M}_{\odot}$, giving the original references and some details on each mass measurement. In the following we discuss briefly these super-massive NSs, and argue that compact binary MSPs are key to establishing what is the maximum NS mass.

Table 1: Super-massive neutron stars, sorted in order of increasing mass measurement/constraint. Compact binary MSPs (spiders) are shown in boldface. Errors are at the 1- $\sigma$ confidence level (ranges indicate mass constraints rather than actual measurements).

\begin{tabular}{lccccc}
\hline \hline Name & Type $^{a}$ & $\mathrm{M}_{\mathrm{NS}}\left(\mathrm{M}_{\odot}\right)$ & Mean error & $\mathrm{P}_{\text {orb }}(\mathrm{hr})$ & Ref. $\left(\mathrm{M}_{\mathrm{NS}}\right)$ \\
\hline \hline PSR J0348+0432 & MSP+WD & $2.01 \pm 0.04$ & $2 \%$ & 2.5 & {$[14]$} \\
3FGL J2039.6-5618 & RBc & $\mathbf{2 . 0 4}+\mathbf{0 . 3 7} b$ & $\mathbf{1 5 \%}$ & $\mathbf{5 . 5}$ & {$[\mathbf{2 0}]$} \\
PSR B1516+02B & $\mathrm{MSP}+\mathrm{WD}$ ? & $1.5-2.2^{c}$ & $19 \%$ & 164.6 & {$[9 ; 46]$} \\
Vela X-1 & $\mathrm{HMXB}$ & $2.12 \pm 0.16$ & $8 \%$ & 215.1 & {$[47]$} \\
PSR J0740+6620 & $\mathrm{MSP}+\mathrm{WD}$ & $2.14_{-0.09}^{+0.10}$ & $4 \%$ & 115.2 & {$[45]$} \\
PSR J1311-3430 & $\mathbf{B W}$ & $\mathbf{1 . 8 - 2 . 7 ^ { d }}$ & $\mathbf{2 0 \%}$ & $\mathbf{1 . 6}$ & {$[\mathbf{4 4}]$} \\
PSR J2215+5135 & RB & $\mathbf{2 . 2 7} \pm \mathbf{0 . 1 6}$ & $\mathbf{7 \%}$ & $\mathbf{4 . 1}$ & {$[\mathbf{1 9}]$} \\
PSR B1957+20 & $\mathbf{B W}$ & $\mathbf{2 . 4 0} \pm \mathbf{0 . 1 2}$ & $\mathbf{5 \%}$ & $\mathbf{9 . 2}$ & {$[\mathbf{1 5}]$} \\
PSR J1748-2021B & $\mathrm{MSP}+\mathrm{WD}$ ? & $2.1-2.9^{e}$ & $15 \%$ & 493.2 & {$[9 ; 48]$} \\
\hline \hline
\end{tabular}

${ }^{a}$ RB: redback; RBc: redback candidate; BW: black widow; HMXB: high-mass X-ray binary; MSP+WD: millisecond pulsar with white dwarf companion (a question mark indicates that this WD identification is uncertain).

${ }^{b}$ Candidate RB, no pulsar detected yet. This $\mathrm{M}_{\mathrm{NS}}$ comes from optical light curve modelling only, without considering radial velocities.

${ }^{c}$ Note this is a probabilistic constraint assuming random inclination. We give latest reported range from Özel \& Freire. Original $1-\sigma$ range was $2.08 \pm 0.19 \mathrm{M}_{\odot}$.

${ }^{d}$ Formally $2.25 \pm 0.45 \mathrm{M}_{\odot}$, but authors give $1.8-2.7 \mathrm{M}_{\odot}$ range from a discussion of possible systematics, so this is a mass constraint (not a $1-\sigma$ range).

${ }^{e}$ Note this is a probabilistic constraint assuming random inclination. We give latest reported range from Özel \& Freire. Original $1-\sigma$ range was $2.74 \pm 0.21 \mathrm{M}_{\odot}$.

As mentioned in Section 1, the first-discovered compact binary MSP [49], the original black widow pulsar PSR B1957+20, holds the current record of the highest NS mass measurement, with $\mathrm{M}_{\mathrm{NS}}=2.40 \pm 0.12 \mathrm{M}_{\odot}$ [15]. As discussed by van Kerkwijk et al., this result may be sensitive to systematic uncertainties on the orbital inclination $\left(\mathrm{i}=65^{\circ} \pm 2\right.$, which the authors take from earlier light curve modelling results; [50]) and on the center of mass velocity (estimated from a combination of simulated spectra and analytical arguments ([15]; see their Section 5). An independent analysis and joint modeling of the light and radial velocity curves, in light of recent results, should be able to establish or update this mass measurement. In their final analysis of the light and radial velocity curves of the black widow PSR J1311-3430, Romani et al. report a NS mass in the range $1.8-2.7 \mathrm{M}_{\odot}$ and argue that the accuracy of this measurement is partly limited by optical flares and variable wind emission lines [44].

The mass of the X-ray pulsar in the high-mass X-ray binary (HMXB) Vela X-1 has also been found to be higher than $2 \mathrm{M}_{\odot}: 2.12 \pm 0.16 \mathrm{M}_{\odot}$ [47]. Even though the optical determination of the companion's velocity may suffer from additional systematics, most measurements indicate that this HMXB hosts a massive NS [51; 52]. In globular clusters, dynamical measurements of the 
companion star are challenging, but there is also evidence for super-massive NSs [48; 46]. Precise long-term timing of radio MSPs in wide eccentric binaries has allowed highly significant detections of the rate of advance of periastron. If this advance is exclusively due to general-relativistic periastron precession, such detections lead to an accurate measurement of the total mass in the binary [16]. Even if the companion is undetected and the orbital inclination is unknown, Freire et al. used probabilistic arguments to constrain the masses of two such pulsars to be close to or above $2 \mathrm{M}_{\odot}$ (PSR J1748-2021B and PSR B1516+02B, see Table 1).

Other redback MSPs have been found that may be close to or above the $2 \mathrm{M}_{\odot}$ limit. Strader and collaborators set a rather high lower limit on the mass of PSR J1048+2339: $\mathrm{M}_{\mathrm{NS}}>1.96 \pm 0.22 \mathrm{M}_{\odot}$ ([20]; as they note, the relatively large uncertainty on this limit can be improved with more precise dynamical studies). The redback candidate 3FGL J2039.6-5618 constitutes a similar case: a measurement close to $2 \mathrm{M}_{\odot}$ but with a relatively large statistical uncertainty $\left(2.04_{-0.25}^{+0.37} \mathrm{M}_{\odot}\right)$. Because pulsations have not been detected and this measurement is based solely on light curve modeling (without radial velocity measurements available so far), the implications for $\mathrm{M}_{N S}^{\max }$ are less certain.

As mentioned in Section 1, Antoniadis and collaborators found a NS mass of $2.01 \pm 0.04 \mathrm{M}_{\odot}$ using pulsar timing and optical observations of PSR J0348+0432 (modeling the WD absorption lines to find $\mathrm{M}_{c}$; see [14]). Most recently, Cromartie et al. [45] found in PSR J0740+6620 another super-massive NS with a mass above $2 \mathrm{M}_{\odot}: \mathrm{M}_{\mathrm{NS}}=2.14 \pm 0.10 \mathrm{M}_{\odot}$. This result comes from a different method to measure $\mathrm{M}_{\mathrm{NS}}$, i.e., the measurement of the relativistic Shapiro delay (see also $[13 ; 53])$. Finding super-massive NSs with independent measurement techniques is important to establish their maximum mass, and to rule out systematic effects in the mass determination (ideally, applying these different techniques to the same pulsar).

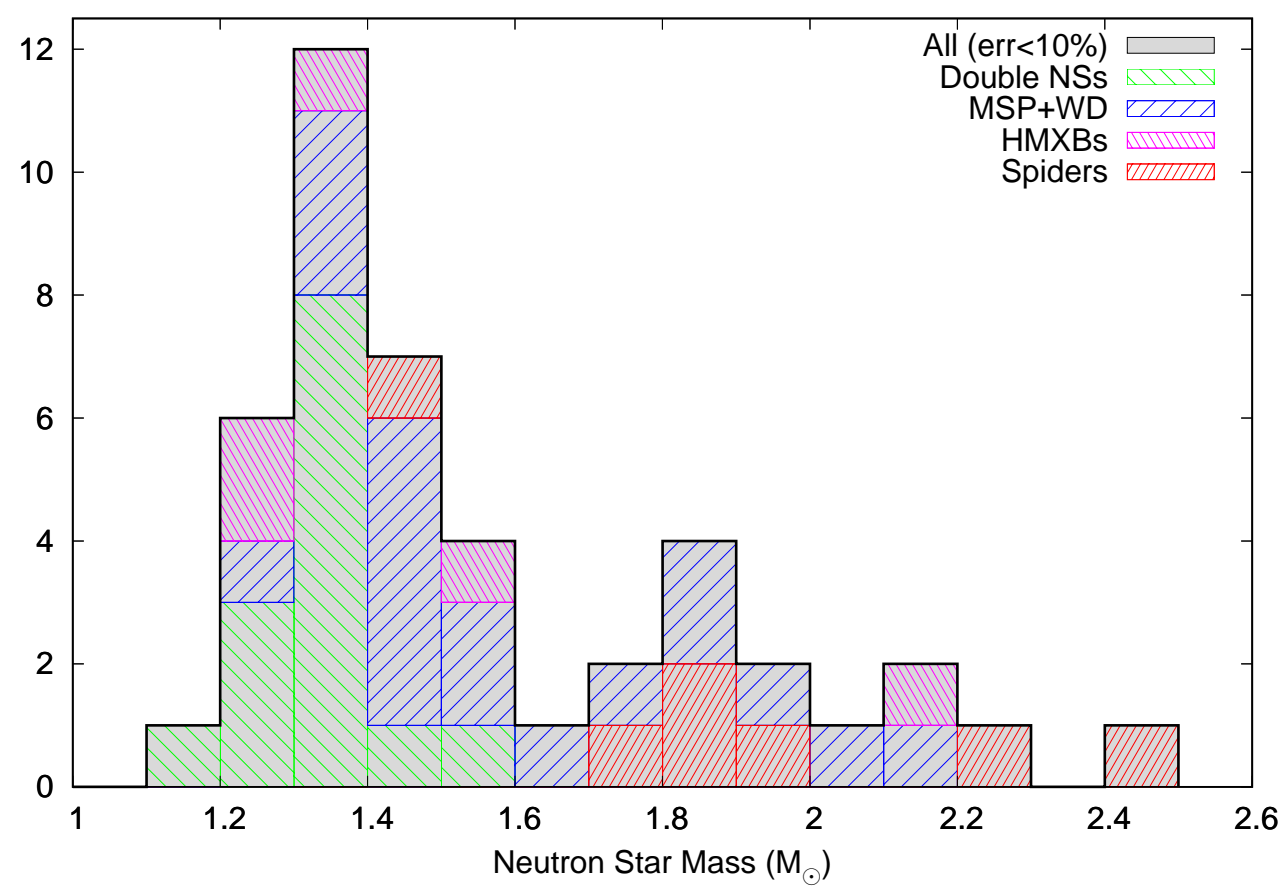

Figure 4: Histogram of the 44 NS mass measurements with errors smaller than $10 \%$, showing the different source classes. While double NSs cluster around $1.35 \mathrm{M}_{\odot}$, spiders have masses from 1.4 to $2.4 \mathrm{M}_{\odot}$. 


\section{Conclusions}

To summarize, we show in Figure 4 a histogram of NS masses, using only mass measurements with fractional uncertainties below 10\% (which includes 44 out of the 86 systems presented in Figure 3). It has been already pointed out that double NSs show a narrow mass distribution centered around $1.35 \mathrm{M}_{\odot}$ and that recycled fast-spinning MSPs are on average more massive [12; 8; 16;9]. During the past decade, we have found mounting evidence for super-massive NSs with masses in the range 2-2.5 $\mathrm{M}_{\odot}$ (Table 1). Here we stress that the growing population of compact binary MSPs shows great potential for finding super-massive NSs and therefore constraining $\mathrm{M}_{N S}^{\max }$ and the EoS of ultradense matter. Indeed, 4 of the 9 systems with potentially super-massive NSs shown in Table 1 are redbacks (one of them candidate) or black widows, and this number may well increase as the population of compact binary MSPs continues to grow. Newly discovered nearby redback MSPs, in particular, hold the greatest potential for the next decade, as they have moderately lowmass companion stars which are sufficiently bright in the optical band (typically $\sim 18-20 \mathrm{mag}$ ) to pursue high S/N spectroscopy and detailed dynamical studies.

\section{References}

[1] J. Casares and P. A. Charles, Optical studies of V404 Cyg, the X-ray transient GS 2023+338. IV. The rotation speed of the companion star., MNRAS 271 (1994) L5.

[2] F. X. Timmes, S. E. Woosley and T. A. Weaver, The Neutron Star and Black Hole Initial Mass Function, ApJ 457 (1996) 834 [astro-ph/9510136].

[3] M. A. Alpar, A. F. Cheng, M. A. Ruderman and J. Shaham, A new class of radio pulsars, Nature 300 (1982) 728.

[4] LiGO SCIENTIFIC COLlaboration AND Virgo Collaboration collaboration, GW170817: Observation of Gravitational Waves from a Binary Neutron Star Inspiral, Phys. Rev. Lett. 119 (2017) 161101.

[5] B. Margalit and B. D. Metzger, Constraining the Maximum Mass of Neutron Stars from Multi-messenger Observations of GW170817, ApJL 850 (2017) L19 [1 710 . 0 5938].

[6] E. Salpeter, Matter at high densities, Annals of Physics 11 (1960) 393.

[7] D. Page and S. Reddy, Dense Matter in Compact Stars: Theoretical Developments and Observational Constraints, Annual Review of Nuclear and Particle Science 56 (2006) 327 [astro-ph/0608360].

[8] J. M. Lattimer and M. Prakash, Neutron star observations: Prognosis for equation of state constraints, PhysRep 442 (2007) 109 [arXiv: astro-ph/ 0612440 ].

[9] F. Özel and P. Freire, Masses, Radii, and the Equation of State of Neutron Stars, ARAA 54 (2016) 401 [1603.02698]. 
[10] R. N. Manchester, G. B. Hobbs, A. Teoh and M. Hobbs, The Australia Telescope National Facility Pulsar Catalogue, AJ 129 (2005) 1993 [astro-ph/ 0412641 ].

[11] D. Lorimer, Galactic Millisecond Pulsars, Online catalog at http://astro.phys.wvu.edu/GalacticMSPs (2019) .

[12] S. E. Thorsett and D. Chakrabarty, Neutron Star Mass Measurements. I. Radio Pulsars, ApJ 512 (1999) 288 [arXiv:astro-ph/9803260].

[13] P. B. Demorest, T. Pennucci, S. M. Ransom, M. S. E. Roberts and J. W. T. Hessels, $\underline{A}$ two-solar-mass neutron star measured using Shapiro delay, Nature 467 (2010) 1081 [1010.5788].

[14] J. Antoniadis, P. C. C. Freire, N. Wex, T. M. Tauris, R. S. Lynch, M. H. van Kerkwijk et al., A Massive Pulsar in a Compact Relativistic Binary, Science 340 (2013) 448 [1304. 6875].

[15] M. H. van Kerkwijk, R. P. Breton and S. R. Kulkarni, Evidence for a Massive Neutron Star from a Radial-velocity Study of the Companion to the Black-widow Pulsar PSR B1957+20, ApJ 728 (2011) 95 [1009.5427].

[16] F. Özel, D. Psaltis, R. Narayan and A. Santos Villarreal, On the Mass Distribution and Birth Masses of Neutron Stars, ApJ 757 (2012) 55 [1201.1006].

[17] R. W. Romani, A. V. Filippenko, J. M. Silverman, S. B. Cenko, J. Greiner, A. Rau et al., PSR J1311-3430: A Heavyweight Neutron Star with a Flyweight Helium Companion, ApJL 760 (2012) L36 [1210 . 6884].

[18] R. P. Breton, M. H. van Kerkwijk, M. S. E. Roberts, J. W. T. Hessels, F. Camilo, M. A. McLaughlin et al., Discovery of the Optical Counterparts to Four Energetic Fermi Millisecond Pulsars, ApJ 769 (2013) 108 [1302 . 1790].

[19] M. Linares, T. Shahbaz and J. Casares, Peering into the Dark Side: Magnesium Lines Establish a Massive Neutron Star in PSR J2215+5135, ApJ 859 (2018) 54 [18 05 . 0879 9].

[20] J. Strader, S. Swihart, L. Chomiuk, A. Bahramian, C. Britt, C. C. Cheung et al., Optical Spectroscopy and Demographics of Redback Millisecond Pulsar Binaries, ApJ 872 (2019) $42[1812.04626]$.

[21] P. Freire, Pulsars in Globular Clusters, Online catalog at http://www.naic.edu/pfreire/GCpsr.html (2019) .

[22] F. Acero et al., Fermi Large Area Telescope Third Source Catalog, ApJS 218 (2015) 23 [1501.02003].

[23] The Fermi-LAT collaboration, Fermi Large Area Telescope Fourth Source Catalog, arXiv e-prints (2019) arXiv:1902.10045 [1902.10045]. 
[24] J. W. T. Hessels, M. S. E. Roberts, M. A. McLaughlin, P. S. Ray, P. Bangale, S. M. Ransom et al., A 350-MHz GBT Survey of 50 Faint Fermi $\gamma$-ray Sources for Radio Millisecond

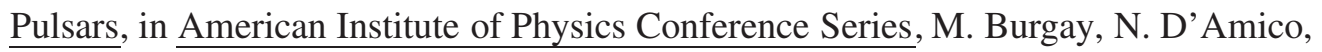
P. Esposito, A. Pellizzoni and A. Possenti, eds., vol. 1357 of American Institute of Physics Conference Series, pp. 40-43, 2011, 1101.1742, DOI.

[25] S. M. Ransom, P. S. Ray, F. Camilo, M. S. E. Roberts, Ö. Çelik, M. T. Wolff et al., Three Millisecond Pulsars in Fermi LAT Unassociated Bright Sources, ApJL 727 (2011) L16 [1012.2862].

[26] S. D. Bates, M. Bailes, N. D. R. Bhat, M. Burgay, S. Burke-Spolaor, N. D'Amico et al., The High Time Resolution Universe Pulsar Survey - II. Discovery of five millisecond pulsars, MNRAS 416 (2011) 2455 [1101 . 4778].

[27] P. S. Ray, A. A. Abdo, D. Parent, D. Bhattacharya, B. Bhattacharyya, F. Camilo et al., Radio Searches of Fermi LAT Sources and Blind Search Pulsars: The Fermi Pulsar Search Consortium, 2011 Fermi Symposium proceedings - eConf C110509; ArXiv 1205.3089 (2012) [1205.3089].

[28] K. Stovall, R. S. Lynch, S. M. Ransom, A. M. Archibald, S. Banaszak, C. M. Biwer et al., The Green Bank Northern Celestial Cap Pulsar Survey. I. Survey Description, Data Analysis, and Initial Results, ApJ 791 (2014) 67 [1406.5214].

[29] F. Camilo, M. Kerr, P. S. Ray, S. M. Ransom, J. Sarkissian, H. T. Cromartie et al., Parkes $\underline{\text { Radio Searches of Fermi Gamma-Ray Sources and Millisecond Pulsar Discoveries, The }}$ Astrophysical Journal 810 (2015) 85 [1507.04451].

[30] S. D. Bates, D. Thornton, M. Bailes, E. Barr, C. G. Bassa, N. D. R. Bhat et al., The High Time Resolution Universe survey - XI. Discovery of five recycled pulsars and the optical detectability of survey white dwarf companions, MNRAS 446 (2015) 4019 [1411. 128 8].

[31] S. Sanpa-Arsa, Searching for New Millisecond Pulsars with the GBT in Fermi Unassociated Sources, PhD Thesis, University of Virginia (2016).

[32] R. W. Romani and M. S. Shaw, The Orbit and Companion of Probable $\gamma$-Ray Pulsar J2339-0533, ApJL 743 (2011) L26 [1111.3074].

[33] A. K. H. Kong, R. H. H. Huang, K. S. Cheng, J. Takata, Y. Yatsu, C. C. Cheung et al., Discovery of an Unidentified Fermi Object as a Black Widow-like Millisecond Pulsar, ApJL 747 (2012) L3 [1201. 3629].

[34] R. W. Romani, 2FGL J1311.7-3429 Joins the Black Widow Club, ApJL 754 (2012) L25 [1207.1736].

[35] R. W. Romani, A. V. Filippenko and S. B. Cenko, 2FGL J1653.6-0159: A New Low in Evaporating Pulsar Binary Periods, ApJL 793 (2014) L20 [1 408 . 28 86]. 
[36] K.-L. Li, A. K. H. Kong, X. Hou, J. Mao, J. Strader, L. Chomiuk et al., Discovery of a Redback Millisecond Pulsar Candidate: 3FGL J0212.1+5320, ApJ 833 (2016) 143 [1609.02951].

[37] M. Linares, P. Miles-Páez, P. Rodríguez-Gil, T. Shahbaz, J. Casares, C. Fariña et al., $\underline{A}$ millisecond pulsar candidate in a 21-h orbit: 3FGL J0212.1+5320, MNRAS 465 (2017) 4602 [1609.02232].

[38] H.-L. Chen, X. Chen, T. M. Tauris and Z. Han, Formation of Black Widows and Redbacks Two Distinct Populations of Eclipsing Binary Millisecond Pulsars, ApJ 775 (2013) 27 [1308.4107].

[39] J. Schroeder and J. Halpern, Observations and Modeling of the Companions of Short Period Binary Millisecond Pulsars: Evidence for High-mass Neutron Stars, ApJ 793 (2014) 78 [1401.7966].

[40] R. W. Romani, M. L. Graham, A. V. Filippenko and M. Kerr, Keck Spectroscopy of Millisecond Pulsar J2215+5135: A Moderate-M $\mathrm{M}_{N S}$, High-inclination Binary, ApJL 809 (2015) L10 [1506.04332].

[41] R. W. Romani and N. Sanchez, Intra-binary Shock Heating of Black Widow Companions, ApJ 828 (2016) 7 [1606.03518].

[42] R. W. Romani, M. L. Graham, A. V. Filippenko and W. Zheng, PSR J1301+0833: A Kinematic Study of a Black-widow Pulsar, ApJ 833 (2016) 138.

[43] T. Shahbaz, M. Linares and R. P. Breton, Properties of the redback millisecond pulsar binary 3FGL J0212.1+5320, MNRAS 472 (2017) 4287 [1708.07355].

[44] R. W. Romani, A. V. Filippenko and S. B. Cenko, A Spectroscopic Study of the Extreme Black Widow PSR J1311-3430, ApJ 804 (2015) 115 [1503.05247].

[45] H. T. Cromartie, E. Fonseca, S. M. Ransom, P. B. Demorest, Z. Arzoumanian, H. Blumer et al., Relativistic Shapiro delay measurements of an extremely massive millisecond pulsar, Nature Astronomy (2019) arXiv:1904.06759 [1904.06759].

[46] P. C. C. Freire, A. Wolszczan, M. van den Berg and J. W. T. Hessels, A Massive Neutron Star in the Globular Cluster M5, ApJ 679 (2008) 1433 [0 712 .3826].

[47] M. Falanga, E. Bozzo, A. Lutovinov, J. M. Bonnet-Bidaud, Y. Fetisova and J. Puls, Ephemeris, orbital decay, and masses of ten eclipsing high-mass X-ray binaries, A\&A 577 (2015) A130 [1502. 07126].

[48] P. C. C. Freire, S. M. Ransom, S. Bégin, I. H. Stairs, J. W. T. Hessels, L. H. Frey et al., Eight New Millisecond Pulsars in NGC 6440 and NGC 6441, ApJ 675 (2008) 670 [0711. 0925].

[49] A. S. Fruchter, D. R. Stinebring and J. H. Taylor, A millisecond pulsar in an eclipsing binary, Nature 333 (1988) 237. 
[50] M. T. Reynolds, P. J. Callanan, A. S. Fruchter, M. A. P. Torres, M. E. Beer and R. A. Gibbons, The light curve of the companion to PSR B1957+20, MNRAS 379 (2007) 1117 [0705.2514].

[51] M. H. van Kerkwijk, J. van Paradijs and E. J. Zuiderwijk, On the masses of neutron stars., A\&A 303 (1995) 497 [astro-ph/ 9505071 ].

[52] H. Quaintrell, A. J. Norton, T. D. C. Ash, P. Roche, B. Willems, T. R. Bedding et al., The mass of the neutron star in Vela X-1 and tidally induced non-radial oscillations in GP Vel, A\&A 401 (2003) 313 [arXiv: astro-ph/0301243].

[53] E. Fonseca, T. T. Pennucci, J. A. Ellis, I. H. Stairs, D. J. Nice, S. M. Ransom et al., The NANOGrav Nine-year Data Set: Mass and Geometric Measurements of Binary Millisecond Pulsars, ApJ 832 (2016) 167 [1603.0 0545$].$

\section{DISCUSSION}

JOSEP MARIA PAREDES: Are the orbital period and the companion star's rotational period synchronized?

MANUEL LINARES: Yes, as a result of tidal forces the orbits are circular and the companion is "tidally locked".

SANDRO MEREGHETTI: a) Is there any correlation between pulsar mass and other system parameters? b) In the case of PSR J2215+5135 (and super-massive NSs in general): how much of that mass has been accreted?

MANUEL LINARES: a) Not that we know of. b) According to evolutionary models (e.g. Chen et al. 2013), redbacks can accrete up to $0.3-0.5 \mathrm{M}_{\odot}$ during their active accretion phase. This suggests that the pulsars would have to be born massive to go beyond $2 M_{\odot}$.

ANDREA SANTANGELO: Some quark-based equations of state for the NS core do allow for super-massive NSs with $\mathrm{M}_{N S}>2 \mathrm{M}_{\odot}$.

MANUEL LINARES: Yes, hyperons seem ruled out but deconfined quarks may be able to do it (especially in the most recent quark EoSs). 